\title{
GESTÃO DA QUALIDADE E DA PRODUÇÃO: INTEGRAÇÃO DE TÉCNICAS AVANÇADAS E SUAS APLICABILIDADES NA INDÚSTRIA MODERNA
}

CLÁUDIO FILIPE LIMA RAPÔSO (engcfraposo@gmail.com) - Mestrando em Administração (IDEIA/Columbia), Pós-Graduado em Gestão em Engenharia de Produção (Instituto Graduarte), MBA em Gestão de Projetos (Instituto Graduarte), Bacharel em Engenharia de Produção (Faculdade Estácio de Sá do Recife) e Bacharelando em Engenharia Agrícola Ambiental (UFRPE).

MARINA LOURENÇO DA SILVA (marinalourenco_@hotmail.com) - Bacharel em Arquitetura e Urbanismo (UFPE).

RESUMO: E A preocupação com a Produção no século XXI é destaque em um mundo cada vez globalizado e as necessidades humanas cada vez mais complexas e insaciáveis. O presente trabalho busca uma abordagem sobre as técnicas Lean Manufacturing, Six Sigma e World Class Manufacturing (WCM) destacando sua importância para o mercado industrial, a busca do conhecimento dessas ferramentas no século XXI. A integração do dessas ferramentas tende a elevar plenamente a qualidade do produto, no processo de produção, na redução de diversos custos, na conservação do ambiente de trabalho e no desenvolvimento de uma indústria mais fluida.

PALAVRAS-CHAVE: Lean Manufacturing, Six Sigma, Lean WCM.

RESUMEN: La preocupación por la producción en el siglo XXI es importante en un mundo cada vez más globalizado y humano necesitan productos cada vez más complejos e insaciable. Este estudio pretende abordar acerca de las técnicas de Lean Manufacturing, Six Sigma y Manufactura World Class Manufacturing (WCM) destacando su importancia para el mercado industrial, la búsqueda del conocimiento de estas herramientas en pleno siglo XXI. La integración de estas herramientas tiende a aumentar plenamente la calidad del producto, en el proceso de producción, reducción de los diversos costos de la conservación del medio ambiente de trabajo y el desarrollo de una industria más fluido.

PALABRAS CLAVES: Lean Manufacturing, Six Sigma, Lean WCM. 


\section{INTRODUÇÃO}

A preocupação com a Produção no século XXI é destaque em um mundo cada vez globalizado e as necessidades humanas cada vez mais complexas e insaciáveis. É um mar estranho e indomável e quem dominá-lo obterá lucros extremos em uma época de crises humanitárias.

Taylor (1966) em sua preocupação entre aos empregados e os lucros empresariais deram início a uma revolução na indústria como conhecemos criando a Administração Cientifica.

Em 1920, Ford (1922) inicia conceito de Produção em Massa tem início em 1910 com Henry Ford, observando o potencial mercado consumidor de classe mais baixa para utilização de carros. Esse produto pela produção artesanal possui custos elevados, o que não agradava as pessoas, Dessa forma, utilizou-se dos conceitos de Taylor para melhorar sua produção, dando início ao Fordismo. (CORREA, H. L. 2004).

Através da cronometragem do trabalho, Taylor (1966), desenvolveu técnicas de melhoramento de tempo e esforço, e formas motivacionais obtendo resultados bastante expressivos para a época.

Outro teórico famoso é o Smith (1982), que desenvolveu a divisão do trabalho em que o funcionário não se preocupava com a construção do produto no geral e sim em partes específicas.

Segundo Machline (1994), essa doutrina foi absorvida pelo Fordismo transformando a forma de produção mais dinâmica e eficiente. Ford conseguiu reduzir o custo de produção consideravelmente, mas um dos maiores defeitos desse sistema de produção é a formação excessiva de estoque.

Em 1950 nasce com Toyota Taiichi o Sistema Toyota de Produção que ao Produzir mais com menos recursos recebeu a alcunha de Produção Enxuta que visa à diminuição de defeitos, excesso de produção, estoques de mercadoria, processamento, movimento e transporte desnecessário e o tempo ocioso na produção. (WERKEMA, M. C. C. 2012).

Ainda segundo o autor, em 1988, após o sucesso da Motorola que pelos seus resultados ganhou o Prêmio Nacional de Qualidade Malcolm Baldrige torna-se público a metodologia Six Sigma que visa a resoluções de problemas não identificados estrategicamente e que gera custos desnecessários na empresa.

Em outra vertente Schonberger, nos anos 80, segundo Ribeiro (2014) apud Flynn (1999) "descreve as sinergias entre qualidade e custo, por exemplo, como o número de defeitos é reduzido através de uma gestão de qualidade, o montante gasto em retrabalho é garantia de reduzido." dando início ao World Class Manufacturing (WCM).

Logo essas técnicas se completam e são aderidas pelas grandes indústrias no mundo. Dessa forma, ao citar essas técnicas o trabalho tem como objetivo uma análise dessas técnicas avançadas e suas aplicabilidades na indústria moderna. 
O presente trabalho busca uma abordagem das seguintes sobre as técnicas Lean Manufacturing, Six Sigma e World Class Manufacturing (WCM) na busca entender a importância para o mercado industrial, a busca do conhecimento dessas ferramentas para o profissional e gestores no século XXI.

\section{MATERIAIS E METODOS}

Através de uma robusta análise bibliográfica, esse trabalho tem como finalidadede fazer um comparativo entre as metodologia Lean Manufacturing, Six Sigma e WCM observadas caracterizando ás semelhanças e diferenças e a integralização das ferramentas aplicadas ao ramo industrial.

\section{LEAN MANUFACTURING}

Nos anos 50, preocupados com as limitações do modelo proposto por Henry Ford e o aumento da exigência dos clientes por produtos diferenciados, nasce o a manufatura Enxuta (Lean Manufacturing) tendo como premissa o alinhamento e sequenciamento da produção com foco em agregar valor à produção. (JONES, D. e WOMACK, J. 1998).

O sistema de Manufatura Enxuta tem como foco principal a diminuição do lead time (tempo que leva para uma peça percorrer todo o caminho no chão de fábrica), sendo necessária a eliminação de todo o tipo de desperdício existente nos processos, através da maximização da produtividade e efetividade dos processos já existentes. [...] A manufatura enxuta é uma forma de tornar o trabalho mais satisfatório, oferecendo feedback imediato sobre os esforços para transformar "muda" (desperdício) em valor, fazendo com que a motivação da força de trabalho seja maior (DIAS, R. L. T. 2006).

Conforme Jones e Womack (1998) agregar valor de forma eficaz era uma das prioridades do Lean Manufacturing, se preocupando em fornecer produtos exatamente solicitados pelo cliente diminuindo o desperdício em valor.

Dias (2006) explica que "Existem alguns princípios que devem ser seguidos pelas organizações para que a manufatura enxuta funcione por completo. Esses conceitos deverão ser seguidos na ordem em que aparecem." O sistema lean se baseia em quatro princípios que são:

- Valor: Definição de o quanto o cliente está disposto a pagar para adquirir produto específico (Bens e/ou Serviços);

- Cadeia de Valor: Esse conceito está em "separar os processos em três tipos: aqueles que efetivamente geram valor, aqueles que não geram valor, mas são importantes para a manutenção da qualidade" (Lean Institute Brasil, 2002);

- Fluxo de Valor: Analise da fluidez do processo e identificação de ociosidade e geração de estoque;

- Produção Puxada: A condução do processo será estimulada pelo cliente de cada fase do processo; (JONES, D. e WOMACK, J. 1998). 
- Perfeição: Todo processo deve está em busca do Melhoramento continuo.

Delfino (2014) apud Slack, Chambers E Johnston (2012) acredita que a união desses objetivos é a eliminação todos os desperdícios de modo a desenvolver uma operação rápida, confiável, que gera produtos de qualidade e custo baixo.

Dessa forma, visando à eliminação de possíveis desperdícios, Jones e Womack (1998) identificaram sete possíveis onde o sistema Lean deve-se trabalhar:

- Superprodução: Produção acima do necessário;

- Tempo de Espera: Tempo oriundo da espera de materiais na produção de estoque no processo;

- Transporte: Movimentação desnecessária do produto no processo;

- Processo: Material desperdiçado no próprio processo por desvio de finalidade e manutenção;

- Estoque: Redução do custo de estocagem;

- Movimentação: Falha no deslocamento do trabalhador no processo;

- Produtos Defeituosos: Produtos fora das especificações de qualidade a perda da função de origem.
Para aplicação do Lean Manufacturing algumas ferramentas são necessárias, dessa forma Werkema (2012) cita: o Mapeamento de Fluxo de Valor, as Métricas Lean, o Kaizen, o Kanban, a Padronização, o 5S, a Redução de Set Up, a Manutenção Produtiva Total, a Gestão Visual e o Poka-Yoke.

\section{SIX SIGMA}

Seis Sigma é uma prática usual nas
grandes empresas, pois traz inúmeras
vantagens a qualquer organização. Sejam
vantagens econômicas, de processo,
ambientais, de qualidade, confiabilidade,
dentre tantas outras, permite à
organização ostentar resultados
significativos na conquista por mercados.
(FIGUEREDO, T. G. 2006).

Essa metodologia estratégica empresarial tem natureza quantitativa e foco no aumento dá à redução das despesas em relação à receita e na melhoria dos resultados. Através dela, busca o melhoramento continuo por resoluções de problemas que não são identificados facilmente.

O sigma citado nessa metodologia representa a medida de variação de uma função normal representada nos processos em gerais. "0 termo Seis Sigma define uma medição da qualidade: 3.4 defeitos por milhão de eventos ou 99,99966\% de perfeição" (FIGUEREDO, T. G. 2006).

A aplicação do Six Sigma é estimulada através da utilização do ciclo DMAIC, sendo cada letra, segundo Werkema (2011): 
- Define: É a definição da situaçãoproblema a ser pesquisado, da equipe e da documentação referente ao projeto. Ferramentas utilizadas: SIPOC e Fluxograma;

- Mensure: Nessa fase será desenvolvido o Plano de coleta e a análise dos dados, tem como objetivo definir quais defeitos influi mais o custo de produção, identificando assim a causa raiz no processo. Utilizam-se frequentemente às seguintes ferramentas, FMEA e SWOT e $5 \mathrm{~W} 2 \mathrm{H}$.

- Analyse: Análise de todos os dados mensurados para definição da causa raiz. Nessa fase utiliza-se bastante o Pareto, Ishikawa e análise de KPI's. Nesse momento é que devem ser apresentadas as melhorias ao Empresário responsável.

- Improve: Através da implementação, deve-se pôr em prática a melhoria no sistema;

- Control: Fase onde será refeita a parte de medição para concretizar as melhores e demonstrar o impacto na empresa, será concluída o projeto e os dados devem ser refeitos periodicamente.

Os projetos de Six sigma possuem uma hierarquização definida e os projetos são mais complexos conforme aumenta o grau de instrução "belts" Werkema (2011): define como:
- Master Black Belt: Assessoram os empresários em busca de projetos de melhoria e coordena os projetos Black Belts;

- Black Belt: Desenvolve projetos estratégicos para a empresa e normalmente que integram diversos setores empresariais;

- Green Belt: São dedicados a setores específicos da empresa e possuem qualidades mais estatísticas de análise do processo;

- Yellow Belt: Auxiliam os Belts na aquisição de dados, possuem conhecimentos mais técnicos sobre a metodologia;

- White Belt: Pessoas que tem o conhecimento da metodologia através de curso de curta duração e superficial, ou possui conhecimentos empíricos sobre a metodologia.

$$
\text { Conforme as necessidades }
$$
empresárias foram ficando mais complexas, houve a necessidade de agregar o Lean Manufacturing na formação do Six Sigma, gerando a metodologia Lean Six Sigma que utiliza das duas ferramentas para solucionar problemas. 


\section{WORD CLASS MANUFACTURING (WCM)}

Campana (2008) define WCM como “[...] um método de gestão que indica e elimina as perdas existentes nos processos produtivos e administrativos, maximiza o uso do ativo industrial e garante a geração de produtos de alta qualidade a custos competitivos".

Tem como objetivo eliminar a causas das quebras e(ou) defeitos, evitando problemas na produção. Visa aumentar a eficácia do maquinário com o auxílio dos trabalhadores baseado na gestão pessoal e no incremento da disponibilidade.

Os pilares que sustentam essa metodologia é direcionada pelo Japan Institute of Plant Maintanace (JIPM), sendo, segundo Campana (2008), Ferreira (2012) e Ribeiro (2014) definidas como:

- Cost Pilar: que busca a redução e otimização de custos por análises confiáveis com intuito de tomada de decisão;

- Supply Chain Pilar: "O propósito do pilar de Supply Chain é realizar a gestão das matérias primas e do produto final do ponto de vista do custo, prazos, quantidades e qualidade acordada com o cliente"; (FERREIRA, S. A. 2012).

- Focused Improvement Pilar: Tem como foco evitar desperdícios de material, de tempo e evitar a ineficiência;
- Quality Maintenance Pilar: Okhovat et al (2012) define esse pilar como a busca a redução dos defeitos até o estado zero.

- Environment Pilar: Esse pilar é específico para questões ambientais, através dele se faz o controle de problemas com o meio ambiente que afetem a empresa e os stakeholders;

- Early Product Management Pillar: Ferreira (2012) explica que Tem como objetivo desenvolver novos produtos através de projetos com a metodologia Designs Reviews;

- Planned Maintenance Pillar: Pilar que é responsável pela manutenção dos equipamentos melhorando a eficiência e diminuindo as horas de perdas;

- Early Equipment Management Pillar: Visa à gestão de projetos avançados, precisos e inovadores focando a melhoria do equipamento;

- Office Pillar: Tem como objetos a otimização contínua dos processos administrativos;

- Safety \& Health Pillar: "O propósito do pilar de Segurança é alcançar e suportar zero acidente e prevenir as doenças ocupacionais através de ferramentas"; (FERREIRA, S. A. 2012). 
- Autonomous Maintenance Pillar: Capacitação dos operadores com o intuito de zelar e assegurar o funcionamento dos equipamentos;

- Education \& Training Pillar: Qualificar os funcionários nas áreas comportamentais, cursos de capacitação e aprimoramento;

- Early Building Management Pillar: elaborar projetos de obras civis pela metodologia Designs Reviews.

Ribeiro (2014) explica que "A estratégia de manufatura deve por sua vez ser ligada com o nível de negócios e planos estratégicos corporativos da empresa." Dessa forma, para a melhor atividade do WCM, deve-se ter apoio da equipe em geral.

Como uma ferramenta avançada de Gestão da Produção, é necessária de forma equivalente a mudança de cultura e aceitação do empregado ao novo sistema, assim como a constante capacitação dos trabalhadores.

Segundo Okhovat et al (2012), essa ferramenta se integra com outras por que em sua estrutura fundamenta-se na mentalidade enxuta, também sendo conhecido como Lean WCM. Em sua estrutura de pilares pode-se incluir internamente o Six Sigma com ferramenta de melhoramento de qualidade, assim como Total Quality Control (TQC).

\section{CONCLUSÃO}

Nesse trabalho podemos evidenciar às funções das três ferramentas e sua aplicabilidade na indústria, conforme o desenvolvimento dessas e sua aplicação no mercado geraram-se a diminuição dos custos e aumento de lucros e melhoria no maquinário interno e condições de trabalho.

O LeanManufacturing integrado ao Six Sigma se torna importante ao mercado por resoluções de problemas mais complexos e de altos custos implícitos na empresa. Além de eliminar o que é descartável para a empresa, tem a capacidade de encontrar problemas na qualidade e "invisíveis" no processo produtivo.

A preocupação do Six Sigma com a eliminação de custos e melhoramento da confiabilidade dos produtos faz parte de vários pilares do WCM. Essa visão faz com que possamos utilizar às ferramentas dentro dos processos de diversos pilares.

A necessidade na filosofia de redução de lead times faz com que a mentalidade enxuta seja base de muitas resoluções de problemas dentro do contexto fabril.

Dessa forma, esse trabalho conclui que apesar de serem distintas as ferramentas, a integração do Lean Manufacturing, Six Sigma e WCM tende a elevar plenamente a qualidade do produto e do processo de produção, a redução de diversos custos, conservação do ambiente de trabalho e o desenvolvimento de uma indústria mais fluida. 


\section{REFERÊNCIAS BIBLIOGRÁFICAS}

- CAMPANA, W. APLICAÇÃO DO CONCEITO DE LEAN MANUFACTURING PARA DESENVOLVIMENTO DE CÉLULA PARA MONTAR MECANISMO LIMPADOR DE PARABRISA. Monografia. São Paulo: USF, 2008.

- CORREA, H. L. Administração da produção e operações - manufatura e serviços: uma abordagem estratégica. São Paulo: Atlas, 2004.

- DELFINO, E. L. de M. O ESTUdo DA PROdUÇÃo ENXUTA NA ELIMINAÇÃO DE DESPERDÍCIOS E SUA APLICAÇÃO EM UMA EMPRESA DE GELADOS COMESTÍVEIS. Monografia. Minas Gerais: UFMG, 2014.

- DIAS, R. L. T. CONCEITOS DE MANUFATURA ENXUTA APLICADOS A UMA INDÚSTRIA DE SUPRIMENTOS E DISPOSITIVOS MÉDICOS. Monografia, Minas Gerais: UFJF, 2006.

- FERREIRA, S.A. EVOLUÇÃO DO GERENCIAMENTO DE PROCESSOS ATRAVÉS DA METODOLOGIA TPM E OS DESAFIOS DO PÓSMPLEMENTAÇÃO EM UMA EMPRESA DE GRANDE PORTE. Monografia. Paraná: UTPR 2012.

- FIGUEREDO, T. G. METOdOLOGIA SEIS SIGMA COMO ESTRATÉGIA PARA REDUÇÃo DE CUSTOS: ESTUDO DE CASO SOBRE A REDUÇÃO DE CONSUMO DE ÓLEO SINTÉTICO NA OPERAÇÃO DE USINAGEM. Monografia. Minas Gerais: UFJF, 2016.

- FLYNN, B. World-class manufacturing project: overview and selected results. International Journal of Operations \& Production Management, United States, 1997.

- FORD, H.. My life and Work.New York: Doubleday, 1922.

- JONES, D. T; WOMACK, J. A Mentalidade Enxuta nas Empresas: elimine os desperdícios e crie riqueza. Rio de Janeiro: Campus. 2009.

- $\quad$ Lean Institute Brasil. Lean Thinking: Conceitos e Aplicações. São Paulo: 2002.

- MACHLINE, C. Evolução da Administração da Produção no Brasil. São Paulo: RAE, v.34, n०3 1994.

- OKHOVAT, M. A. et al. Development of world class manufacturing framework by using six-sigma, total productive maintenance and lean. Scientific Research and Essays Vol. 7: 2012.

- RIBEIRO, A. P. UTILIZAÇÃO DA MANUFATURA DE CLASSE MUNDIAL (WCM) COMO UMA FERRAMENTA ESTRATÉGICA DE DIFERENCIAÇÃO COMPETITIVA. Monografia. Paraná: UTPR 2014. 
- SLACK, N; CHAMBERS, S; JOHNSTON, R. Administração da Produção. $3^{a}$ Ed. São Paulo: Editora Atlas S.A., 2009.

- SMITH, A. The Wealth of Nations. New York: Penguin Books, 1982.

- TAYLOR, F. W. Princípios de Administração Científica. São Paulo: Atlas, 1966.

- WERKEMA, C. Lean Seis sigma: Introdução às ferramentas do Lean Manufacturing. 2. ed. Minas Gerais: Elsevier, 2012.

- WERKEMA, C. Lean Seis sigma. 1. ed. Minas Gerais: Elsevier, 2011.

\section{NOTAS BIBLIOGRÁFICAS}

\section{Cláudio Filipe Lima Rapôso}

Mestrando em Administração (IDEIA/Columbia), Pós Graduado em Gestão em Engenharia de Produção (Instituto Graduarte), MBA em Gestão de Projetos (Instituto Graduarte), Bacharel em Engenharia de Produção (Faculdade Estácio de Sá do Recife) e Bacharelando em Engenharia Agrícola Ambiental (UFRPE). Participante das equipes Campus Party Recife (2012 à 2016), Palestrante sobre Gestão Industrial, Gestão da Qualidade, Gestão de Projetos, Gestão de Equipe e Empreendedorismo. Professor Autônomo de Excel Avançado, Microsoft Project e Primavera Project. Ex-Estagiário na empresa Petróleo Brasileiro SIA (PETROBRAS), Ex-Bolsista PIBIC no Laboratório Lasaq e Genoma (UFRPE) e CEO da Lourenço Raposo Consultoria MEI.

\section{Marina Lourenço da Silva}

Bacharel em Arquitetura e Urbanismo (UFPE). 